\title{
Cerebellar-Parietal Connections Underpin Phonological Storage
}

\author{
Katja Macher, ${ }^{1}$ Andreas Böhringer, ${ }^{2}$ Arno Villringer, ${ }^{1,3,4}$ and Burkhard Pleger ${ }^{1,4}$ \\ ${ }^{1}$ Department of Neurology, Max Planck Institute for Human Cognitive and Brain Sciences, 04103 Leipzig, Germany, ${ }^{2}$ Clinic for Psychiatry and \\ Psychotherapy, Central Institute for Mental Health, 68159 Mannheim, Germany, ${ }^{3}$ Berlin School of Mind and Brain, Mind and Brain Institute, 10099 Berlin, \\ Germany, and ${ }^{4}$ Clinic for Cognitive Neurology, University Hospital Leipzig, 04103 Leipzig, Germany
}

Previous research has accumulated convincing evidence to show that the human cerebellum contributes to the short-term storage of verbal information, but its specific role in brain networks involved in phonological storage remains uncertain. In a randomized, crossover and sham-controlled design, we here combined transcranial direct current stimulation (tDCS), applied to the right cerebellum, with fMRI to investigate systematically the contribution of the human cerebellum to encoding, maintenance, and retrieval of verbal information. After anodal, but not cathodal, tDCS, we found a reduced item recognition capacity together with an attenuated neural signal from the right cerebellar lobule VIIb, specifically during the late encoding phase. Within this phase, tDCS furthermore affected task-associated functional connections between right cerebellar lobule VIIb and the posterior parietal cortex. These findings suggest that the right cerebellar lobule VIIb interacts with the posterior parietal cortex, specifically during the late stages of verbal encoding, when verbal information enters phonological storage.

Key words: brain connectivity; cerebellum; posterior parietal cortex; functional magnetic resonance imaging; noninvasive brain stimulation; verbal working memory

\section{Introduction}

Several lines of research suggest that the cerebellum contributes to cognitive processes such as attention shifting, perception, memory, and even emotional processing (Ravizza et al., 2006; Kirschen et al., 2010; Stoodley et al., 2010). One of the cognitive faculties involving the cerebellum is verbal working memory. Desmond et al. (Desmond et al., 1997; Chen and Desmond, 2005a), intensively studied the cerebellum's contribution to the related cognitive demands. Based on their fMRI studies showing different coactivations between cerebral and cerebellar regions, they formulated a human cerebrocerebellar verbal working memory model that is supported by the cerebrocerebellar network architecture of primates.

These animal studies identified corticopontine projections from frontal regions that are distributed along medial portions of the pontine nuclei (Brodal, 1978; Wiesendanger et al., 1979; Leichnetz et al., 1984; Schmahmann, 1996; Schmahmann and Pandya, 1997a, 1997b), which in turn project to superior cerebel-

\footnotetext{
Received Jan. 10, 2014; revised Feb. 14, 2014; accepted March 4, 2014.

Author contributions: K.M., A.B., and B.P. designed research; K.M. performed research; A.B. and A.V. contributed unpublished reagents/analytic tools; K.M. and B.P. analyzed data; K.M. and B.P. wrote the paper.

This work was supported by the Federal Ministry of Education and Research (Bernstein Focus, State Dependencies of Learning 01G00975, Project 18GL4DW4 to B.P.).

The authors declare no competing financial interests.

Correspondence should be addressed to either of the following: Burkhard Pleger, Clinic for Cognitive Neurology, University Hospital Leipzig, Liebigstraße 16, 04103 Leipzig, Germany, E-mail: bpleger@cbs.mpg.de; or Katja Macher, Max Planck Institute for Human Cognitive and Brain Sciences, Department of Cognitive Neurology, Stephanstr. 1a, 04103 Leipzig, Germany, E-mail: macher@cbs.mpg.de.

DOI:10.1523/JNEUROSCI.0106-14.2014

Copyright $\odot 2014$ the authors $\quad 0270-6474 / 14 / 345029-09 \$ 15.00 / 0$
}

lar areas, including lobule VI and Crus I (Brodal, 1979, 1982; Glickstein et al., 1994). Consistent with these anatomical observations in primates, Desmond et al. (Desmond et al., 1997; Chen and Desmond, 2005a) found that, in humans, Broca's area coactivated with cerebellar lobule VI/Crus I directly after visual presentation of the verbal items that subjects had to remember. Based on these findings, they postulated that projections between Broca's areas and the superior portions of the cerebellum may underpin articulatory control processes.

Neuroanatomical research in primates furthermore unveiled connections between parietal regions and lateral pontine regions (Brodal, 1978; Wiesendanger et al., 1979; Leichnetz et al., 1984; Weber and Yin, 1984; May and Andersen, 1986; Schmahmann and Pandya, 1989, 1991; Schmahmann, 1996; Brodal and Bjaalie, 1997), which project to the cerebellar paramedian lobule VIIb (Brodal, 1979, 1982; Glickstein et al., 1994). Analogously to these findings, Desmond et al. (Desmond et al., 1997; Chen and Desmond, 2005a) found hemodynamic coactivations between parietal regions and right cerebellar lobule VIIb, specifically during phonological storage processes. The strict rightsidedness of this latter cerebellar activation in lobule VIIb fits the known cerebrocerebellar cross-lateralization, which implies that, just as the left hemisphere is involved in language processing, the right cerebellum is involved in language-based functions (Chen and Desmond, 2005a, 2005b; Friederici, 2011).

Previous studies either aimed at the identification of taskrelated cerebellar activation (Desmond et al., 1997; Chen and Desmond, 2005a) or used noninvasive cerebellar stimulation to prove its causal role in verbal short-term memory (Ferrucci et al., 2008; Boehringer et al., 2013). What remains speculative is the 
cerebellum's specific role in different cognitive subprocesses, for example, as described by Desmond's cerebrocerebellar model (Desmond et al., 1997; Chen and Desmond, 2005a). To this end, we here used noninvasive cerebellar transcranial direct current stimulation (tDCS) to modulate an individual's verbal memory capacity together with fMRI to capture altered activity either during encoding, late encoding, maintenance, or retrieval of verbal information within right cerebellar subregions that were previously reported to be involved (i.e., lobules VI, VIIIa/b, VIIa, CrusI, VIIb; Desmond et al., 1997; Chen and Desmond, 2005a).

\section{Materials and Methods}

Subjects

We recruited 16 young, healthy, right-handed volunteers ( 8 females, age $26 \pm 3.4$ years). All participants gave written informed consent and the study protocol was approved by the ethics committee of the medical faculty of the University of Leipzig.

\section{Experimental schedule}

We used a sham-controlled, randomized, crossover design. The order of tDCS conditions was counterbalanced across subjects. For each of the three tDCS conditions (sham, anodal, and cathodal tDCS), subjects were invited to attend our laboratory on two successive days (Fig. 1A). On the first day, they underwent two training sessions for the Sternberg task (Sternberg, 1966), which we modified for the purposes of this study (see Modified Sternberg item recognition fMRI task, below, for further details) outside of the MRI scanner to establish stable baseline performance. There was a $25 \mathrm{~min}$ break between the two sessions. On the second day, subjects first completed one session of the Sternberg task (i.e., presession) before we applied one of the three tDCS protocols over the right cerebellar hemisphere. After tDCS application, participants were again engaged in the Sternberg task, but this time during brain imaging (i.e., fMRI postsession; Fig. $1 A$ ).

\section{Modified Sternberg item recognition $\mathrm{fMRI}$ task}

In our fMRI variant of Sternberg's item recognition task, each trial consisted of the known four different phases as already implemented in the original version (Sternberg, 1966), each demanding different cognitive skills (Fig. $1 B$ ). First, participants had to encode the acoustically presented set of digits (i.e., encoding phase). In the first three seconds of the maintenance phase, encoding is paralleled by storage processes (i.e., late encoding phase; Leung et al., 2004; Postle, 2006). In the maintenance phase, which follows the late encoding phase, meaning is elaborated and implemented to the semantic network (Craik et al., 2000). In the fourth and last phase (i.e., retrieval phase), participants had to retrieve the memorized verbal information from phonological storage (Rypma and D'Esposito, 1999).

We here modified Sternberg's version of the task by adapting its difficulty to each subject's individual memory capacities. This modification allowed inferences not solely based on reaction times (D'Esposito et al., 1998), but also on the number of recognized digits (i.e., memory capacity), because tDCS-induced changes in reaction times may simply occur because of a tDCS-induced interference with the known cerebellar motor control functions such as timing (Ito, 2008).

In our previous tDCS studies, we used digit forward and backward spans together with tDCS to investigate the cerebellum's causal role in verbal short-term memory processes (Boehringer et al., 2013). Because our digit span experiments showed a significant influence of cathodal tDCS on memory recall (Boehringer et al., 2013), we decided to design the tDCS/fMRI experiments accordingly. To this end, we applied tDCS before subjects were engaged in the task using the same stimulation parameters and even the same device (see tDCS, below, for more details). For the same reason, we decided to modify the original Sternberg task and to present the digits acoustically instead of visually.

In two brief extra sessions before the first training sessions, we tested subject's individual memory capacity using a staircase procedure by adding one digit per trial. If the subjects failed to recognize the target digit on two successive trials, the staircase was stopped. We computed the median of recognized digits across both sessions. For the following sessions, we used three difficulty levels centered on this median (i.e., median, median minus five digits, median plus five digits). The variation by five digits was derived from pilot experiments involving nine other subjects who performed the Sternberg task outside of the MRI scanner. The task consisted of four sessions and two runs for each subject. We computed the SD to the median across sessions, which was five digits. Based on these pilot experiments, we adjusted the difficulty levels to the median plus and minus five digits.

Digits were presented acoustically via speakers (training and pre scanning sessions) or MRI-compatible headphones (fMRI session) at a rate of one digit per second (Fig. 1B). After a break of $7 \mathrm{~s}$, the target digit was acoustically presented and subjects had to decide via a button press whether it was part of the set of digits or not. Trials were presented in blocks of two trials of the same difficulty level (i.e., same amount of digits). The intertrial interval between trials (and blocks) was $4 \mathrm{~s}$. The order of blocks was pseudorandomized over each session. Trial difficulty was visually cued for $2 \mathrm{~s}$ before each block. Seven seconds after the presentation of the set of digits, the target digit was presented acoustically and an "X" appeared on the screen. When the "X" appeared, subjects had to indicate via button press whether the target digit had been part of the foregoing set of digits. The target digit was part of this sequence in $50 \%$ of all trials. 


\section{Statistical analyses of behavioral data}

To analyze the influence of tDCS on reaction times and the number of recognized digits, we used a $3 \times 3$ factorial ANOVA for repeated measures, with the factors pre-post difference either of the reaction times or number of recognized digits for each difficulty level (median minus five digits, median, median plus five digits) and stimulation condition (anodal, cathodal, or sham tDCS). Post hoc paired $t$ tests comparing pretDCS with post-tDCS performance were used to further investigate the structure of significance.

\section{$t D C S$}

tDCS was applied with a pair of surface-soaked sponge electrodes $(5 \times 5$ $\mathrm{cm}$ ) using a commercial tDCS device (NeuroConn). A constant current of $2 \mathrm{~mA}$ (current density $0.08 \mathrm{~mA} / \mathrm{cm}^{2}$ ) was applied to the right cerebellar hemisphere over a period of $25 \mathrm{~min}$. In the anodal stimulation condition, the anode was placed $2 \mathrm{~cm}$ below the inion and $1 \mathrm{~cm}$ posterior to the right mastoid process (Ferrucci et al., 2008) to target the lobules located in the lateral and more caudal parts of the cerebellum (also see Introduction for our a priori hypothesis and the accordingly defined ROI). The cathode was placed over the right musculus buccinator (Galea et al., 2009) for an almost right-angled orientation of the current in relation to the cerebellar surface. For cathodal stimulation, anode and cathode were placed contrariwise. During the sham tDCS condition, the constant current of $2 \mathrm{~mA}$ was applied according to common procedure for only $30 \mathrm{~s}$ before being switched off (Gandiga et al., 2006). We used a stopwatch to guarantee that the fMRI experiment started 7 min after tDCS application.

\section{$f M R I$}

Image acquisition. MRI data were acquired with a Siemens Tim Trio 3T scanner. For functional blood oxygenation level-dependent (BOLD) imaging, we used a T2-sensitive echoplanar imaging sequence (EPI). We acquired 720 EPI volumes with each fMRI session, covering the entire brain (data matrix $64 \times 64, \mathrm{TR}=2000 \mathrm{~ms}, \mathrm{TE}=30 \mathrm{~ms}$, flip angle $=90^{\circ}$, bandwidth $1817 \mathrm{kHz}, \mathrm{FOV}=1152 \mathrm{~mm}$, in-plane resolution $3 \times 3 \mathrm{~mm}$, slice thickness $4 \mathrm{~mm}$, interslice gap $5 \mathrm{~mm}$ ). The first three images were implemented to guarantee a saturated BOLD signal and were discarded from further analyses. Structural images from each subject (T1-weighted $3 \mathrm{D}$ magnetization-prepared rapid gradient echo images $=650 \mathrm{~ms}$, $\mathrm{TR}=$ $1300 \mathrm{~ms}, \mathrm{FOV}=256 \times 240 \mathrm{~mm}, \alpha=10^{\circ}$, two acquisitions, $1 \mathrm{~mm}$ isotropic resolution) were coregistered to the first scan of the functional scan series to superimpose individual brain functions on the underlying brain anatomy.

Preprocessing of $f M R I$ data. For preprocessing and postprocessing of fMRI data, we used SPM8 (Wellcome Department of Imaging Neuroscience, London, UK). According to common procedures, EPI scans were first realigned using the six-parameter affine transformation and unwarped using the $\mathrm{B}=0$ field map scan to account for movements during scanning. Next, images were normalized to Montreal Neurological Institute (MNI) standard space and spatially smoothed with a Gaussian kernel of $6 \mathrm{~mm}$ full width at half maximum.

\section{Statistical analyses}

We used the general linear model (GLM) to assess hemodynamic responses for each task's phase/event (i.e., encoding phase, late encoding phase, maintenance phase, recognition phase) of any given trial. On the single-subject level, each GLM consisted of three sessions (sham, anodal, cathodal tDCS) and each session of 12 regressors capturing the 12 different conditions (four trial phases by three difficulty levels). The onset of each event was convolved with the standard hemodynamic response function (hrf) as implemented in SPM8. For the encoding phase, we first applied a box-car function before convolution with the hrf to account for the different trial lengths because the higher the difficulty level or the better the participant, the longer the sequence of acoustically presented digits. The correlation between regressors after the convolution process was as follows: $r=0.065, \mathrm{SD}=0.046$ (encoding vs late encoding), $r=$ $0.385, \mathrm{SD}=0.035$ (late encoding vs maintenance), $r=0.161, \mathrm{SD}=0.031$ (maintenance vs retrieval), $r=-0.296, \mathrm{SD}=0.009$ (retrieval vs encoding), indicating relatively low covariance for the purpose of assessing phase-specific hemodynamic effects. For the assessment of significant activation within the part of the right cerebellum that was previously shown to be involved in processes underpinning verbal working memory (i.e., lobules VI, VIIa/b, VIIIa/b, CrusI; see Introduction and Desmond et al., 1997; Chen and Desmond, 2005a), we restricted our search volume to the anatomical territory of these cerebellar areas (i.e., small volume correction). To this end, we created a mask including lobules VI, VIIa/b, VIIIa/b, CrusI based on the probabilistic cerebellar atlas by Diedrichsen et al. (2009) as implemented in the Anatomy Toolbox for SPM8 (Eickhoff et al., 2005).

Assessment of task-related phase-specific neural activity. To assess brain activation specifically related to each single phase (or event) of the Sternberg task, we implemented participants' fMRI data acquired after sham tDCS in the SPM8 full factorial design and compared each task phase (i.e., encoding, late encoding, maintenance) with the remaining phases with the exception of the retrieval phase because this phase, compared with the other exclusively cognitive phases of the task (i.e., encoding, late encoding, maintenance), was heavily confounded by motor activity due to the button press used by participants to indicate their decision (Fig. 3, last row). To assess neural activation related to the retrieval phase, we compared this phase with the remaining phases (i.e., encoding, late encoding, maintenance).

Assessment of $t D C S$-induced alterations in cerebellar activity. In accordance with the influence of tDCS on subjects' behavior (i.e., reduced/ increased number of recognized digits for any of the three difficulty levels), we investigated tDCS influences on neural cerebellar activity. To this end, we used the paired $t$ test to compare fMRI data acquired after sham tDCS with either anodal or cathodal tDCS (corresponding to one of the two polarities that significantly influenced digit recognition; i.e., same contrast as for the assessment of significant tDCS-induced behavioral alterations) for the different phases of the Sternberg task (i.e., encoding, late encoding, maintenance, retrieval), searching for significantly enhanced or lowered hemodynamic responses in the right cerebellar regions of interest (i.e., lobules VI, VIIa/b, VIIIa/b, CrusI, see Statistical analyses, above, for further details on small volume correction). The significance level was set to $p=0.05$ (familywise error [FWE] corrected).

Assessment of phase-specific tDCS-induced alterations in cerebrocerebellar functional connectivity. To assess the task-related functional connectivity of those cerebellar areas that showed an altered neural signal after tDCS application, we applied the method of psychophysiological interaction (PPI; Friston et al., 1997). On the single-subject level, we first extracted the first Eigenvariate of the task-related neural activation from those cerebellar voxels that were significantly (i.e., $p=0.05$, FWE corrected) affected by either anodal or cathodal tDCS (see previous paragraph for how we assessed tDCS effects within the right cerebellum). This was done for the sham tDCS condition and for the tDCS condition with significantly affected cerebellar activity (i.e., either anodal or cathodal tDCS). For each of the two tDCS conditions, the PPI accounted for phase-specific activation (i.e., comparing the task phase in which we found the tDCS effect- either encoding, late encoding, maintenance, or retrieval, see also previous paragraph for further specifications of the phase-specific contrast- to the remaining phases). On the single-subject level, we computed each of the two PPIs using a GLM model consisting of the task regressors and the PPI regressor that captured the interaction between task conditions and cerebellar activity. On the group level, we applied the paired $t$ test to compare the PPI of the sham tDCS condition with the tDCS condition that significantly affected cerebellar activity (i.e., either anodal or cathodal tDCS). The significance level for the PPI analysis was set to $p=0.001$ (uncorrected).

Association between verbal item recognition capacity and neural activation from the cerebellum and connected brain area(s). To investigate a possible relationship between the individual item recognition capacity and neural activation from either the cerebellar region that was affected by tDCS or the brain area(s) found to be connected to this cerebellar region, we performed Pearson's linear correlation analyses. We implemented the first Eigenvariate across significantly activated voxels after sham tDCS (i.e., no influence of brain stimulation on cerebellar activity) together with the percentage of recognized items in a correlation analysis and tested for either a positive or negative brain-behavioral relationship. 


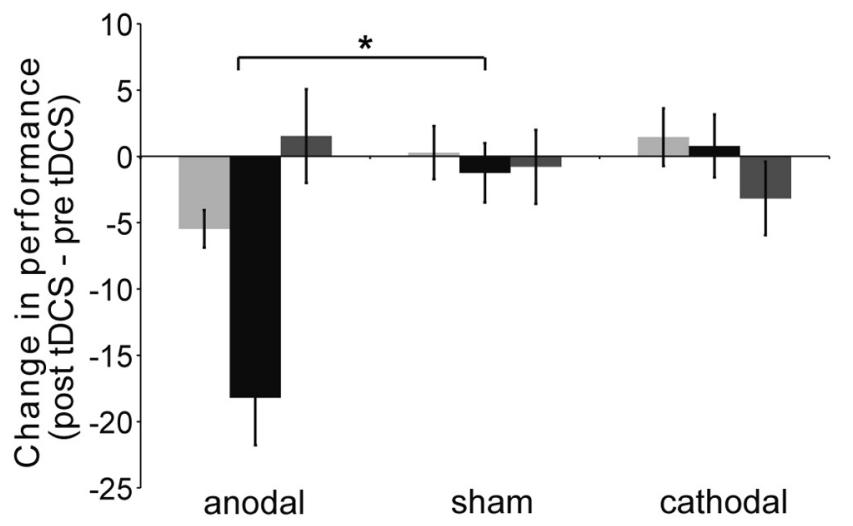

- low difficulty (median capacity - 5 digits)

- medium difficulty (median capacity)

- high difficulty (median capacity +5 digits)

Figure 2. Changes in digit recognition due to cerebellar tDCS (post-tDCS - pre-tDCS) for the three difficulty levels (i.e., high, medium, low) across the three tDCS conditions (i.e., anodal, sham, cathodal). Only after anodal tDCS and only on the medium difficulty level did we find an impaired digit recognition compared with sham tDCS (paired $t$ test comparing sham with anodal tDCS condition $p=0.02$, corrected for multiple comparisons). Error bars indicate SEM.

To assess whether the tDCS-induced alterations in memory capacity correlated with the tDCS-induced alterations in neural activity, we computed pre-post differences of recognized items and the difference in neural activity from the right cerebellum (i.e., the first Eigenvariate from sham tDCS minus the tDCS condition that significantly affected cerebellar function) or area(s) found to be connected to the right cerebellum. The differences in behavioral performance and the differences in neural activity were together implemented in a Pearson correlation analysis to again test for either a positive or negative brain-behavioral relationship.

\section{Results}

\section{Statistical analyses of behavioral data}

The $3 \times 3$ factorial ANOVA ( 3 task difficulty levels $\times 3$ tDCS conditions) of reaction times revealed a main effect for task difficulty $\left(F_{(2,30)}=17.89, p<0.001\right)$, but no effect with respect to the tDCS conditions $\left(F_{(2,30)}=0.80, p=0.923\right)$, suggesting that cerebellar tDCS had only negligible influences on subjects' motor responses.

The same analysis, but with pre-post differences of recognized digits, revealed a main effect of tDCS condition $\left(F_{(2,30)}=4.35\right.$, $p<0.05)$ and an interaction of tDCS and task difficulty $\left(F_{(4,60)}=\right.$ $3.45, p<0.05$ ) (Fig. 2). Post hoc paired $t$ tests comparing pre$\mathrm{tDCS}$ with post-tDCS performance revealed a reduced digit recognition capacity only after anodal tDCS and only on the medium difficulty level $\left(t_{(15)}=2.56\right.$; $\left.p<0.05\right)$, but not on the lowest (median minus 5 digits: $t_{(15)}=1.94$; n.s.) or highest level (median plus 5 digits: $t_{(15)}=-0.27$; n.s). There was also no significant effect for sham or cathodal tDCS for any of the three difficulty levels.

\section{Functional brain imaging}

Assessment of task-related phase-specific neural activity

Comparing the encoding phase with the remaining phases (except the retrieval phase, see Materials and Methods for further details on how we assessed phase-specific neural activity), we found a predominant bilateral activity of the auditory cortices located in the middle and superior temporal gyrus (note that digits were acoustically presented; Fig. 3, first row) next to some other regions that are listed in Table 1.

For the late encoding phase, we identified significant activations in the posterior parietal cortex, insula, the supplementary motor area (SMA), the precuneus, the inferior frontal gyrus, the right parahippocampus, the inferior/middle occipital gyrus, the superior/medial/inferior frontal gyrus, the superior/middle temporal gyrus, the precentral gyrus, the middle cingulate gyrus, and the cerebellar lobules VIIIa and VIIb (Fig. 3, second row, Table 1).

For the maintenance phase, we found significant activity in the anterior/ventral inferior prefrontal gyrus (BA 47), which was previously shown to be involved in verbal working memory (Kapur et al., 1994; Demb et al., 1995; Ranganath et al., 2003) and in phonological and semantic processing (McDermott et al., 2003; De Carli et al., 2007; Friederici and Gierhan, 2013). Positioned next to BA 47, we found significant activity in the superior temporal gyrus (Fig. 3, third row; see Table 1 for the complete list of significantly activated brain areas).

The retrieval phase was heavily confounded by motor activation due to the button press used by subjects to indicate their decision. Accordingly, we found an activation pattern that predominantly encompassed motor regions such as the primary motor cortex on the left hemisphere (note that subjects pressed the button with their right index finger), the premotor cortex, the SMA, the inferior/superior occipital gyrus, postcentral gyrus, and motor-associated regions within the cerebellum (Fig. 3, fourth row) positioned next to some other regions that are listed in Table 1.

Table 1 summarizes the peak voxels' MNI coordinates, T scores, cluster sizes, and anatomical assignments of all significantly activated brain areas for each of the four task phases (i.e., encoding, late encoding, maintenance, and retrieval).

\section{Assessment of tDCS-induced alterations in cerebellar activity}

Because we found an effect of tDCS on digit recognition only for anodal tDCS and only on the medium difficulty level (Fig. 2 ), we next looked for corresponding tDCS influences on cerebellar function. On the medium difficulty level, we systematically compared fMRI data acquired after sham tDCS with anodal tDCS for the different phases of the Sternberg task (i.e., encoding, late encoding, maintenance, retrieval), searching for significantly altered hemodynamic responses from the right cerebellar lobules VI, VIIa/b, VIIIa/b, CrusI (i.e., $p<$ 0.05, FWE corrected, see Materials and Methods for further details on small volume correction). We found significantly lowered hemodynamic responses in one right cerebellar area, namely lobule VIIb, which was exclusively evident in the late encoding phase (peak voxel: $x=24, y=-73, z=-47$, $\mathrm{T}=$ 5.48; Fig. $4 A$ ), suggesting that anodal tDCS attenuated cerebellar lobule VIIb activity specifically during this phase. Note that this cerebellar cluster of attenuated neural activity substantially overlapped with the phase-specific cerebellar activation we found for the late encoding phase (Fig. 4A, Fig. 3, second row), underlining the assumption that anodal tDCS attenuated the function of that cerebellar region that was specifically involved in the late encoding of verbal information.

Consistent with the lack of tDCS influences on digit recognition when applied with inversed polarity (i.e., cathodal tDCS), we found no significant increase or decrease in hemodynamic activity in the cerebellum for any of the task's phases after cathodal tDCS.

\section{Assessment of phase-specific tDCS-induced alterations in} cerebrocerebellar functional connectivity

The paired $t$ test (i.e., sham compared with anodal tDCS) of the phase-specific PPIs (i.e., accounting for neural activity specifically related to the late encoding phase, see Materials and Meth- 


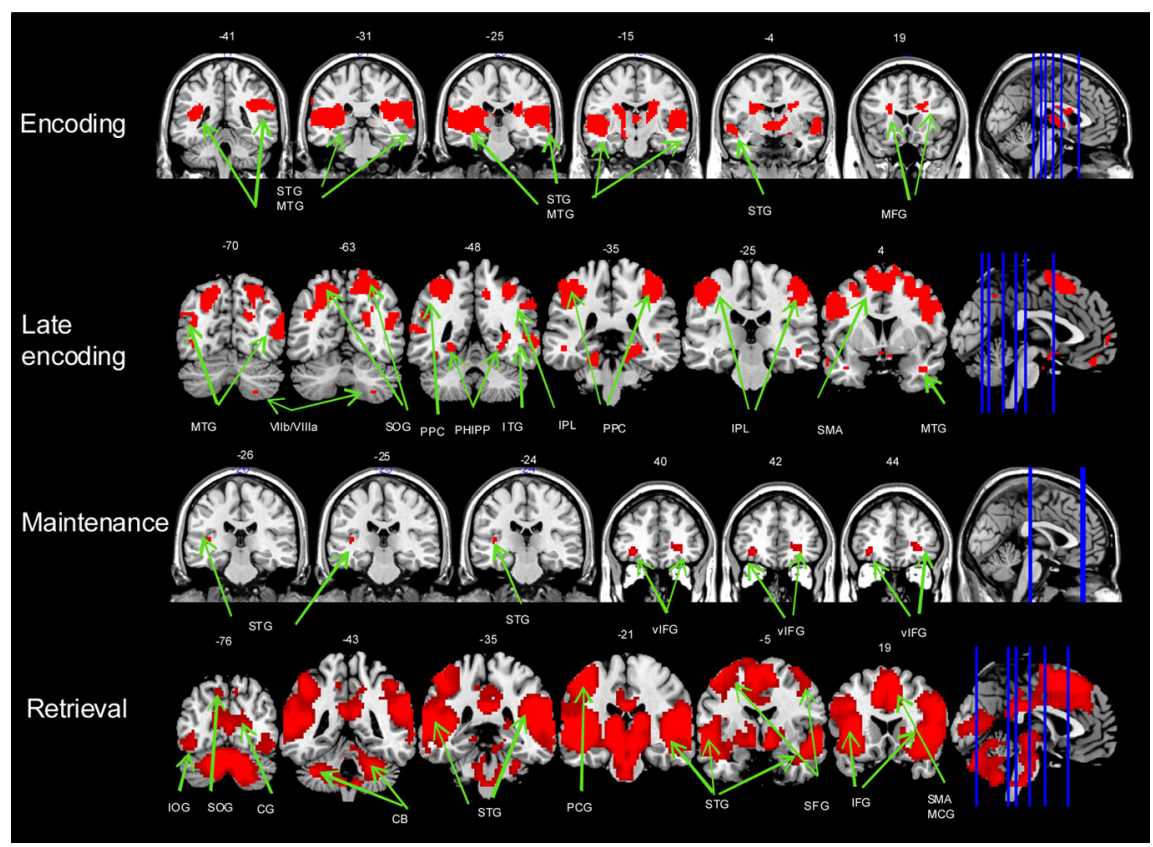

Figure 3. Comparison of four different phases of the Sternberg task. First row, Encoding phase versus late encoding und maintenance phase ( $p=0.005$, uncorrected). Second row, Late encoding phase versus encoding und maintenance phase ( $p=$ 0.005 , uncorrected). Third row, Maintenance versus encoding phase and late encoding phase ( $p=0.005$, uncorrected). Bottom row, Retrieval phase versus encoding, late encoding, maintenance phase ( $p=0.001$, FWE corrected). CB, cerebellum; $C G$, calcarine gyrus; IFG, inferior frontal gyrus; INS, insula; IOG, inferior occipital gyrus; IPL, inferior parietal lobule; ITG, inferior tempora gyrus; MCG, middle cingulate gyrus; MFG, middle frontal gyrus; MTG, middle temporal gyrus; PCG, postcentral gyrus; PHIPP, parahippocampus; PPC, posterior parietal cortex; PREC, precuneus; SOG, superior occipital gyrus; SPL, superior parietal lobule; STG, superior temporal gyrus; vIFG, ventral inferferior frontal gyrus.

ods for further details) seeded on the cerebellar representation of the tDCS effect (i.e., attenuated neural signal caused by anodal tDCS) revealed a tDCS-induced weakening of task-associated functional connectivity between the right cerebellar lobule VIIb and the posterior parietal cortex (peak voxel: $x=-24, y=-49$, $z=67, \mathrm{~T}=5.05$; Fig. $4 B$ ).

Association between verbal item recognition capacity and neural activation from the cerebellum and connected brain area(s) We found a significant positive correlation between neural activation from cerebellar lobule VIIb and the item recognition capacity after sham tDCS $(r=0.658, p=0.006$; Fig. $4 C)$, suggesting that the individual activity level in cerebellar lobule VIIb during the late encoding phase in fact related to the individual memory capacity. Accordingly, the higher the neural activity in cerebellar lobule VIIb, the higher the amount of recognized items. After anodal tDCS was applied, this positive relationship was no longer present $(r=0.038, p=0.888)$. In addition, the difference in item recognition capacity (preperformance - postperformance) showed no relationship with the difference in neural signal from the cerebellar lobule VIIb (first Eigenvariate for sham - anodal tDCS; $r=-0.056, p=0.837)$.

For the posterior parietal cortex, the brain area that we found to be connected to the cerebellar lobule VIIb specifically during the late encoding phase, we found no correlation with the item recognition capacity after sham $\mathrm{tDCS}$ (i.e., during the late encoding phase; $r=0.01, p=0.971$ ) and also no correlation between the parietal cortex, as assessed by PPI analysis (first Eigenvariate for sham - anodal tDCS), and the reduction in item recognition capacity (preperformance - postperformance; $r=-0.005, p=$ $0.985)$.

\section{Discussion}

Our present findings indicate that only anodal cerebellar tDCS causes an attenuated memory recognition capacity. Only after anodal tDCS did we also find significantly attenuated hemodynamic signals from the stimulated right cerebellum, namely from lobule VIIb, together with a weakened functional connectivity of this area with the posterior parietal cortex, specifically during the late encoding phase (Leung et al., 2004; Postle, 2006).

\section{Cerebellar tDCS in cognitive neuroscience}

Most results showing how tDCS affects brain function come from studies in which tDCS was used to modulate motor cortex excitability. These studies suggest that anodal tDCS has a facilitatory influence, whereas cathodal tDCS exerts an inhibitory influence (Nitsche and Paulus, 2000; Lang et al., 2005; Butler et al., 2013). If reduced hemodynamic signal reflects inhibition of cortical excitability, then this taxonomy obviously does not translate to the present findings. However, previous cerebellar tDCS studies also found an inhibitory effect on memory capacity that was independent of whether tDCS was applied with anodal or cathodal polarity (Ferrucci et al., 2008; Boehringer et al., 2013). Based on these inhibitory effects, Ferrucci and Priori (2014) recently hypothesized that cerebellar tDCS may generally interfere with Purkinje cell long-term depression by altering the fine-tuning of membrane potential and the relative pace-making properties. The present findings confirm the inhibitory effect of anodal tDCS. What remains less clear is why we did not find any significant effects caused by cathodal tDCS.

Previously, we showed that cathodal tDCS impaired digit spans (Boehringer et al., 2013: note that we did not assess the effects of anodal tDCS in this previous study), whereas in the present study, we found no effect for cathodal tDCS, but a reduced recognition capacity after anodal tDCS. Because tDCS was applied before the task in both studies with the same parameters and even the same device, the most plausible explanation for the different findings must be the different cognitive processes involved. Although testing the digit span demands recall of memorized digits, the Sternberg task only requires their recognition. Given that, in a recent metaanalysis, cathodal tDCS in combination with cognitive tasks proved a significant lower effect size compared with anodal tDCS (Jacobson et al., 2012), it could well be that the lower cognitive engagement demanded by the Sternberg task (recognition instead of memory recall) and the less effective polarity are together responsible for the lack of effects that we found after cathodal tDCS. In addition to these different polarity-related tDCS effects, we also found that both the cerebellar and behavioral effect were only present on the medium difficulty level, which suggests that tDCS was only effective if subjects were adequately engaged and not when the task was 
Table 1. Significantly activated brain areas

\begin{tabular}{|c|c|c|c|c|c|c|}
\hline Hemisphere & $x$ & $y$ & $z$ & $T$ & Cluster size (voxels) & Location \\
\hline \multicolumn{7}{|c|}{ Encoding phase versus late encoding and maintenance ( $p=0.005$, uncorrected) } \\
\hline Left & -51 & -16 & 1 & 11.44 & \multirow[t]{2}{*}{3443} & Superior temporal gyrus \\
\hline Left & -39 & -28 & 10 & 11.40 & & Superior temporal gyrus \\
\hline \multicolumn{7}{|c|}{ Late encoding versus encoding and maintenance ( $p=0.005$, uncorrected) } \\
\hline Left & -33 & 23 & 4 & 5.14 & 398 & Insula lobe \\
\hline Left & -3 & 62 & 4 & 3.96 & 85 & Superior medial gyrus \\
\hline Left & -12 & -43 & 28 & 3.43 & 15 & Posterior cingulate gyrus \\
\hline Left & -51 & -40 & -5 & 3.42 & 15 & Middle temporal gyrus \\
\hline Left & -57 & -13 & -20 & 3.31 & 15 & Middle temporal gyrus \\
\hline Left & -45 & 47 & -5 & 3.25 & 5 & Middle orbital gyrus \\
\hline Left & -57 & 5 & -20 & 3.23 & 13 & Middle temporal gyrus \\
\hline Left & -36 & -55 & -14 & 2.88 & 12 & Fusiform gyrus \\
\hline Right & 48 & -37 & 7 & 3.47 & 18 & Superior temporal gyrus \\
\hline Right & 15 & -88 & -5 & 3.44 & 16 & Lingual gyrus \\
\hline Right & 57 & -4 & -23 & 3.38 & 22 & Middle temporal gyrus \\
\hline Right & 6 & 20 & -14 & 3.29 & 12 & Olfactory cortex \\
\hline Right & 48 & -25 & -11 & 3.16 & 13 & Fusiform gyrus \\
\hline Right & 9 & -10 & 34 & 3.07 & 5 & Middle cingulate gyrus \\
\hline Right & 12 & -46 & 25 & 3.06 & 9 & Posterior cingulate gyrus \\
\hline Right & 27 & -64 & -50 & 2.97 & 12 & Posterior cingulate gyrus \\
\hline Right & 0 & 47 & -20 & 2.89 & 33 & Rectal gyrus \\
\hline \multicolumn{7}{|c|}{ Maintenance versus encoding and late encoding ( $p=0.005$, uncorrected) } \\
\hline Left & -33 & 41 & -8 & 3.83 & 42 & Middle orbital gyrus \\
\hline Left & -15 & 5 & 25 & 3.71 & 20 & Corpus callosum \\
\hline
\end{tabular}

too difficult or too easy. Based on these findings, we assume a close interaction between tDCS polarity and the specific taskrelated cognitive demand. This assumption is supported by another tDCS study providing additional evidence that tDCS polarity interacts differently with the strength of cognitive engagement (Jones and Berryhill, 2012).

\section{Influence of cerebellar tDCS during late encoding}

To investigate whether anodal tDCS specifically impaired memory encoding, late encoding, maintenance, or retrieval, we assessed phase-specific hemodynamic cerebrocerebellar responses (for further details, see Results, Fig. 1B, Table 1). When systematically testing for a significant tDCS-induced cerebellar effect, we found that anodal tDCS had a specific effect on the first $3 \mathrm{~s}$ of the maintenance phase, when verbal items enter phonological storage (Leung et al., 2004; Postle, 2006). These processes seem to involve the right cerebellum and, more specifically, the posteriorly localized lobule VIIb, which in previous studies was shown to coactivate with phonological storage processes (Chen and Desmond, 2005a, 2005b). This assumption agrees with the present findings and is further supported by the tDCS-induced attenuation of the task-related functional connectivity between cerebellar lobule
VIIb and a cerebral area that is also well known for its involvement in the manipulation and storage of verbal information, the posterior parietal cortex (Collette et al., 1999; Honey et al., 2000).

\section{Influence of cerebellar tDCS on cerebellar-cerebral functional connectivity}

Anodal tDCS applied to the right cerebellar hemisphere weakened the connectivity between right cerebellar lobule VIIb und the posterior parietal cortex (Fig. 4B). These findings agree with the assumption that cerebellar tDCS acts on cerebral structures through cerebellar efferent projections (Ferrucci and Priori, 2014). Furthermore, they emphasize the notion that the posterior parietal cortex acts as a site of phonological storage in verbal working memory processes. This assumption agrees with previous fMRI studies by Chen and Desmond (2005a), who investigated human subjects engaged either in the Sternberg's verbal working memory task or a pure motoric rehearsal task not involving any storage demands. Both tasks provoked considerable activations in the left inferior frontal gyrus, corresponding to Broca's area, as well as bilaterally in the superior cerebellar hemisphere in lobule VI/Crus I. Contrarily, activation in the posterior parietal lobule and supra- 


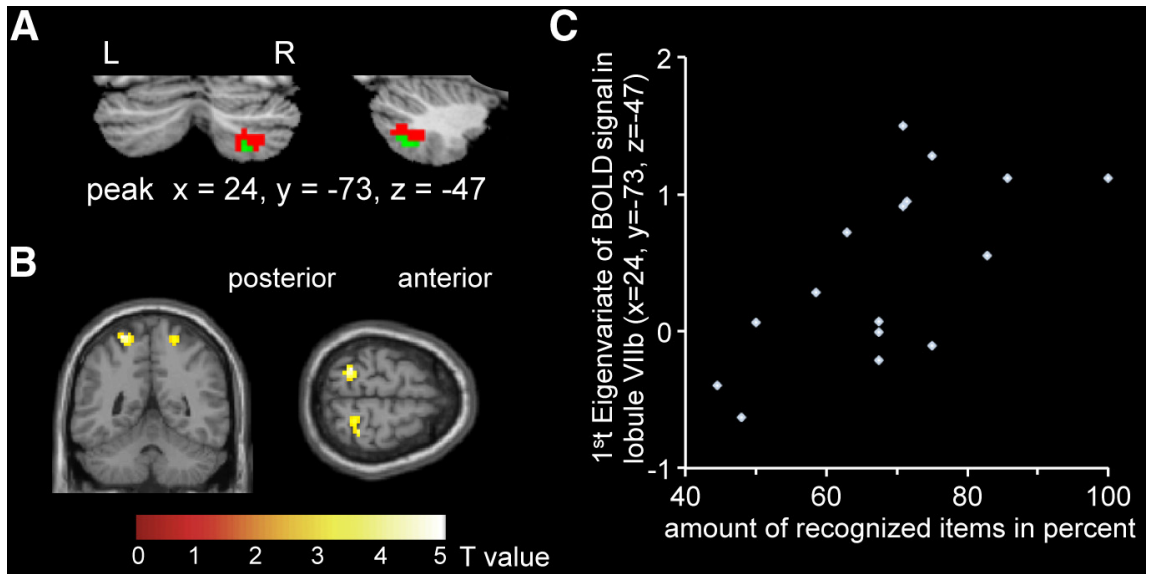

Figure 4. A, After anodal tDCS was applied to the right cerebellum, we found significantly reduced neural activity in right cerebellar lobule VIIb (i.e., red cluster of voxels) compared with sham tDCS on the medium difficulty level during the late encoding phase (paired $t$ test comparing sham vs anodal tDCS condition, $p=0.05$, FWE small volume corrected; for display purposes, $p=$ 0.001 , uncorrected; also see Fig. 2 for related tDCS effects on item recognition capacity). Left, Coronal slice. Right, Sagittal slice, $y=$ -73 . Please note the substantial overlap between the effect of anodal tDCS (paired $t$ test comparing sham vs anodal tDCS condition, colored in red) and the cerebellar activity that we found during the late encoding phase (full factorial design comparing late encoding vs the encoding und maintenance phase, colored in green; for display purposes, late-encoding related activity thresholded at $p=0.005$, uncorrected). $\boldsymbol{B}$, PPI seeded on the effect of anodal tDCS in right cerebellar lobule VIlb, which captures the interaction between task conditions and cerebellar activity, yielded a significant task-associated connectivity to the posterior parietal cortex. The stronger functional connectivity after sham tDCS compared with anodal tDCS suggests that anodal tDCS weakened the connections between cerebellar lobule VIIb and the posterior parietal cortex specifically during the late encoding phase (paired $t$ test comparing sham vs anodal $\mathrm{tDCS}, p=0.001$, uncorrected). C, Correlation between first Eigenvariate extracted from right cerebellar lobule VIlb $(x=24, y=-73, z=-47)$ across significantly activated voxels after sham tDCS (i.e., no influence of brain stimulation on cerebellar activity) with the percentage of recognized items $(r=0.658, p=0.006)$. This relationship suggests that the higher the individual activity level in lobule VIllb, the higher the recognition capacity. This correlation underlines the assumption that lobule VIlb underpins phonological storage processes.

marginal gyrus, as well as in the inferior cerebellar lobule VIIb, was observed only for working memory processes. Based on the assumption that articulation is a common component of motoric rehearsal and phonological storage, whereas phonological storage is required only for the working memory processes, Chen and Desmond (2005a) postulated that the superior cerebellar activation together with activity in Broca's area, which are common to both tasks, reflects the articulatory component, whereas the inferior cerebellar activation together with activity in the posterior parietal lobule and supramarginal gyrus, observed only during verbal working memory, reflects phonological storage.

The present fMRI and functional connectivity findings underline the assumption that cerebrocerebellar projections between lobule VIIb and posterior parietal cortex are involved in phonological storage processes. Contrarily, we could not confirm the a priori expected activation in cerebellar lobule VI/ Crus I or in Broca's area, suggesting that the articulatory control process played only a minor or negligible role in our fMRI version of the Sternberg task. The most plausible explanation for the missing activation in this latter cerebrocerebellar connection is the striking difference between the Sternberg tasks used by Chen and Desmond (2005a) and our version. Chen and Desmond (2005a) presented the verbal items visually, whereas we presented them acoustically to keep crucial experimental parameters consistent with our previous tDCS experiments (Boehringer et al., 2013). In our "acoustic" version of the task, the path toward phonological storage may have bypassed subvocal rehearsal processes necessary for translating the visual content into verbal content, which may explain the lack of activation that we found for the corre- sponding cerebrocerebellar projections from Broca's area to lobule VI/Crus I in the superior cerebellar hemisphere. This assumption agrees with Baddeley's model of the phonological loop, suggesting that speech inputs enter the phonological short-term store directly, whereas nonspeech inputs are first subjected to subvocal rehearsal (Baddeley, 1986).

\section{Associations between recognition capacity and cerebrocerebellar activity and connectivity}

The influence of anodal cerebellar tDCS on participants' recognition capacity and on the individual level of cerebellar hemodynamic activity together suggest that the right cerebellar lobule VIIb is involved in processes underpinning verbal working memory. These findings are further supported by the positive correlation we found between both measures. Accordingly, in the control condition (i.e., after sham tDCS), participants with stronger activity in cerebellar lobule VIIb also recognized more items, which alternatively can be interpreted as a possible involvement of cerebellar lobule VIIb in item recognition or, in other words, in retrieval instead of storage processes (Fig. 4C). Our phase-specific fMRI findings nevertheless point to an effect of anodal tDCS not on memory retrieval, but on late encoding processes, suggesting that lobule VIIb rather supports phonological storage. Assuming undisturbed cerebellar function during phonological storage processes (i.e., sham tDCS) and also no disturbances of the processes bridging late encoding with memory retrieval (i.e., maintenance of the stored items), storage capacity should equal recognition capacity. Therefore, the positive brain-behavioral relationship that we found between recognition capacity and cerebellar activity also supports both our assumption and the assumption made by Chen and Desmond (2005a) that the cerebellar lobule VIIb underpins phonological storage processes.

Contrarily, after anodal tDCS, neither cerebellar lobule VIIb nor the connected posterior parietal cortex yielded a relationship with the reduced item recognition capacity. Although it is generally problematic to interpret such nonsignificant findings, which may still become significant with an increase in sample size, the lack of correlation between the tDCS-induced reduction in item recognition capacity and attenuation of neural activity from either the cerebellum or posterior parietal cortex suggests that the effect of anodal tDCS on recognition capacity is not solely related to the altered signal and connections of these brain regions, but potentially also by more distant tDCS effects on other relevant areas within the brain network underpinning either the storage or retrieval of verbal information.

\section{Conclusion}

Combining tDCS with $\mathrm{AMRI}$, we confirm here previous speculations on a specific cerebellar contribution to late encoding pro- 
cesses (Chen and Desmond, 2005a). These findings are supported by the tDCS-induced attenuated functional connectivity between right cerebellar lobule VIIb and the posterior parietal cortex, suggesting that this cerebellar-posterior parietal connection is crucial for processes underpinning phonological storage.

\section{References}

Baddeley AD (1986) Working memory. Oxford: OUP.

Boehringer A, Macher K, Dukart J, Villringer A, Pleger B (2013) Cerebellar transcranial direct current stimulation modulates verbal working memory. Brain Stimul 6:649-653. CrossRef Medline

Brodal P (1978) Principles of organization of the monkey corticopontine projection. Brain Res 148:214-218. CrossRef Medline

Brodal P (1979) The pontocerebellar projection in the rhesus monkey: an experimental study with retrograde axonal transport of horseradish peroxidase. J Neurosci 4:193-208. CrossRef Medline

Brodal P (1982) Further observations on the cerebellar projections from the pontine nuclei and the nucleus reticularis tegmenti pontis in the rhesus monkey. J Comp Neurol 204:44-55. CrossRef Medline

Brodal P, Bjaalie JG (1997) Salient anatomic features of the cortico-pontocerebellar pathway. Prog Brain Res 114:227-249. CrossRef Medline

Butler AJ, Shuster M, O'Hara E, Hurley K, Middlebrooks D, Guilkey K (2013) A meta-analysis of the efficacy of anodal transcranial direct current stimulation for upper limb motor recovery in stroke survivors. J Hand Ther 26:162-170; quiz 171. CrossRef Medline

Chen SH, Desmond JE (2005a) Cerebrocerebellar networks during articulatory rehearsal and verbal working memory tasks. Neuroimage 24:332338. CrossRef Medline

Chen SH, Desmond JE (2005b) Temporal dynamics of cerebro-cerebellar network recruitment during a cognitive task. Neuropsychologia 43:12271237. CrossRef Medline

Collette F, Salmon E, Van der Linden M, Chicherio C, Belleville S, Degueldre C, Delfiore G, Franck G (1999) Regional brain activity during tasks devoted to the central executive of working memory. Brain Res Cogn Brain Res 7:411-417. CrossRef Medline

Craik FI, Naveh-Benjamin M, Ishaik G, Anderson ND (2000) Divided attention during encoding and retrieval: differential control effects? J Exp Psychol Learn Mem Cogn 26:1744-1749. CrossRef Medline

De Carli D, Garreffa G, Colonnese C, Giulietti G, Labruna L, Briselli E, Ken S, Macrì MA, Maraviglia B (2007) Identification of activated regions during a language task. Magn Reson Imaging 25:933-938. CrossRef Medline

Demb JB, Desmond JE, Wagner AD, Vaidya CJ, Glover GH, Gabrieli JD (1995) Semantic encoding and retrieval in the left inferior prefrontal cortex: a functional MRI study of task difficulty and process specificity. J Neurosci 15:5870-5878. Medline

Desmond JE, Gabrieli JD, Wagner AD, Ginier BL, Glover GH (1997) Lobular patterns of cerebellar activation in verbal working-memory and finger-tapping tasks as revealed by functional MRI. J Neurosci 17:96759685. Medline

D’Esposito M, Ballard D, Aguirre GK, Zarahn E (1998) Human prefrontal cortex is not specific for working memory: a functional MRI study. Neuroimage 8:274-282. CrossRef Medline

Diedrichsen J, Balsters JH, Flavell J, Cussans E, Ramnani N (2009) A probabilistic MR atlas of the human cerebellum. Neuroimage 46:39-46. CrossRef Medline

Eickhoff SB, Stephan KE, Mohlberg H, Grefkes C, Fink GR, Amunts K, Zilles K (2005) A new SPM toolbox for combining probabilistic cytoarchitectonic maps and functional imaging data. Neuroimage 25:1325-1335. CrossRef Medline

Ferrucci R, Priori A (2014) Transcranial cerebellar direct current stimulation (tcDCS): Motor control, cognition, learning and emotions. Neuroimage 85:918-923. CrossRef Medline

Ferrucci R, Marceglia S, Vergari M, Cogiamanian F, Mrakic-Sposta S, Mameli F, Zago S, Barbieri S, Priori A (2008) Cerebellar transcranial direct current stimulation impairs the practice-dependent proficiency increase in working memory. J Cogn Neurosci 20:1687-1697. CrossRef Medline

Friederici AD (2011) The brain basis of language processing: from structure to function. Physiol Rev 91:1357-1392. CrossRef Medline
Friederici AD, Gierhan SM (2013) The language network. Curr Opin Neurobiol 23:250-254. CrossRef Medline

Friston KJ, Buechel C, Fink GR, Morris J, Rolls E, Dolan RJ (1997) Psychophysiological and modulatory interactions in neuroimaging. Neuroimage 229:218-229. CrossRef Medline

Galea JM, Jayaram G, Ajagbe L, Celnik P (2009) Modulation of cerebellar excitability by polarity-specific noninvasive direct current stimulation. J Neurosci 29:9115-9122. CrossRef Medline

Gandiga PC, Hummel FC, Cohen LG (2006) Transcranial DC stimulation (tDCS): a tool for double-blind sham-controlled clinical studies in brain stimulation. Clin Neurophysiol 117:845-850. CrossRef Medline

Glickstein M, Gerrits N, Kralj-Hans I, Mercier B, Stein J, Voogd J (1994) Visual pontocerebellar projections in the macaque. J Comp Neurol 349: 51-72. CrossRef Medline

Honey GD, Bullmore ET, Sharma T (2000) Prolonged reaction time to a verbal working memory task predicts increased power of posterior parietal cortical activation. Neuroimage 12:495-503. CrossRef Medline

Ito M (2008) Control of mental activities by internal models in the cerebellum. Nat Rev Neurosci 9:304-313. CrossRef Medline

Jacobson L, Koslowsky M, Lavidor M (2012) tDCS polarity effects in motor and cognitive domains: a meta-analytical review. Exp Brain Res 216:1-10. CrossRef Medline

Jones KT, Berryhill ME (2012) Parietal contributions to visual working memory depend on task difficulty. Front Psychiatry 3:81. CrossRef Medline

Kapur S, Craik FI, Tulving E, Wilson AA, Houle S, Brown GM (1994) Neuroanatomical correlates of encoding in episodic memory: levels of processing effect. Proc Natl Acad Sci U S A 91:2008-2011. CrossRef Medline

Kirschen MP, Chen SH, Desmond JE (2010) Modality specific cerebrocerebellar activations in verbal working memory: an fMRI study. Behav Neurol 23:51-63. CrossRef Medline

Lang N, Siebner HR, Ward NS, Lee L, Nitsche MA, Paulus W, Rothwell JC, Lemon RN, Frackowiak RS (2005) How does transcranial DC stimulation of the primary motor cortex alter regional neuronal activity in the human brain? Eur J Neurosci 22:495-504. CrossRef Medline

Leichnetz GR, Smith DJ, Spencer RF (1984) Cortical projections to the paramedian tegmental and basilar pons in the monkey. J Comp Neurol $388-408$.

Leung HC, Seelig D, Gore JC (2004) The effect of memory load on cortical activity in the spatial working memory circuit. Cogn Affect Behav Neurosci 4:553-563. CrossRef Medline

May JG, Andersen RA (1986) Different patterns of corticopontine projections from separate cortical fields within the inferior parietal lobule and dorsal prelunate gyrus of the macaque. Exp Brain Res 63:265-278. Medline

McDermott KB, Petersen SE, Watson JM, Ojemann JG (2003) A procedure for identifying regions preferentially activated by attention to semantic and phonological relations using functional magnetic resonance imaging. Neuropsychologia 41:293-303. CrossRef Medline

Nitsche MA, Paulus W (2000) Excitability changes induced in the human motor cortex by weak transcranial direct current stimulation. J Physiol 527:633-639. CrossRef Medline

Postle BR (2006) Working memory as an emergent property of the mind and brain. Neuroscience 139:23-38. CrossRef Medline

Ranganath C, Johnson MK, D'Esposito M (2003) Prefrontal activity associated with working memory and episodic long-term memory. Neuropsychologia 41:378-389. CrossRef Medline

Ravizza S, McCormick CA, Schlerf JE, Justus T, Ivry RB, Fiez JA (2006) Cerebellar damage produces selective deficits in verbal working memory. Brain 129:306-320. CrossRef Medline

Rypma B, D’Esposito M (1999) The roles of prefrontal brain regions in components of working memory: effects of memory load and individual differences. Proc Natl Acad Sci U S A 96:6558-6563. CrossRef Medline

Schmahmann JD (1996) From movement to thought: anatomic substrates 
of the cerebellar contribution to cognitive processing. Hum Brain Mapp 4:174-198. CrossRef Medline

Schmahmann JD, Pandya DN (1989) Anatomical investigation of projections to the basis pontis from posterior parietal association cortices in rhesus monkey. J Comp Neurol 289:53-73. CrossRef Medline

Schmahmann JD, Pandya DN (1991) Projections to the basis pontis from the superior temporal sulcus and superior temporal region in the rhesus monkey. J Comp Neurol 308:224-248. CrossRef Medline

Schmahmann JD, Pandya DN (1997a) Anatomic organization of the basilar pontine projections from prefrontal cortices in rhesus monkey. J Neurosci 17:438-458. Medline

Schmahmann JD, Pandya DN (1997b) The cerebrocerebellar system. Int Rev Neurobiol 41:31-60. CrossRef Medline
Sternberg S (1966) High-speed scanning in human memory. Science 153: 652-654. CrossRef Medline

Stoodley CJ, Valera EM, Schmahmann JD (2010) An fMRI study of intraindividual functional topography in the human cerebellum. Behav Neurol 23:65-79. CrossRef Medline

Weber JT, Yin TC (1984) Subcortical projections of the inferior parietal cortex (area 7) in the stump-tailed monkey. J Comp Neurol 224:206-230. CrossRef Medline

Wiesendanger R, Wiesendanger M, Rüegg DG (1979) An anatomical investigation of the corticopontaine projection in the primate (Macaca fascicularis and Saimiri sciureus). II. The projection from frontal and parental association areas. Neuroscience 4:747-765. CrossRef Medline 